【研究简报】

\title{
Fourier 变换系统中相位恢复的递推方法
}

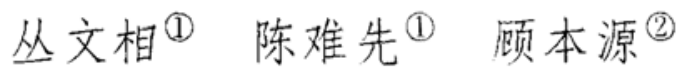 \\ (1)北京科技大学应用物理研究所, 北京 100083; (2)中国科学院物理研究所, 北京 100080)
}

\section{关键词 分数 Fourier 变换 相位恢复 递推算法}

Fourier 变换系统中的相位恢复问题在天文学、衍射光学、电子显微学、X 射线晶体学、全 息成像以及逆源问题等领域都有重要应用 ${ }^{[1]}$. 在实际问题中直接测量的数据常常只是波场 的强度分布, 而波场的相位分布往往很难直接测量, 甚至是不可能的. 因此, 从强度测量数据 来恢复相位分布的问题一直受到人们广泛的关注.

Fourier 变换系统中的相位恢复问题就是用已知输入平面波函数 $f(x)$ 的模 $|f(x)|$ 和输 出平面波函数 $F(u)$ 的模 $|F(u)|$ 重构函数 $f(x)$ (或 $F(u)$ ), 其中 $F(u)$ 是 $f(x)$ 的 Fourier 变 换, 即

$$
F(u)=\int_{-\infty}^{\infty} f(x) \mathrm{e}^{-2 \pi j u x} \mathrm{~d} x .
$$

1971 年 Gerchberg 和 Saxton 首次提出了一种实用的迭代算法 (GS 算法) ${ }^{[2]}$. 后来在 GS 算法基础上, Fienup, Misell, Broucher, 杨国桢和顾本源等 ${ }^{[3,4]}$ 又提出了诸如误差减少法、最速 下降法、共轭梯度法、输入输出法及杨-顾法等多种算法, 进一步改进了 GS 算法. 为了避免迭 代算法计算量过大, 并解决收玫性依赖于初始相位分布选择的问题, Dallas ${ }^{[5]}$ 和 Huiser ${ }^{[6]}$ 提出 了直接方法计算相位分布, 但每步计算中存在的多解选择问题没能很好地解决.

本文将分数 Fourier 变换 (FRFT $)^{[7,8]}$ 应用于 Fourier 变换光学成像系统中的相位恢复问 题, 提出了由强度数据恢复相位分布的一种递推算法.

\section{1 分数 Fourier 变换}

\section{1 分数 Fourier 变换的基本概念 $[9,10]$}

设 $\{F g\}(x)$ 表示 $g(x)$ 的 Fourier 变换, 算子 $F$ 的 $k$ 次幂 $\mathscr{F}^{k}$ 定义为连续 $k$ 次执行算子 那么 $\left\{\mathscr{F}^{2} g\right\}(x)=g(-x)$ 及 $\left\{\mathscr{F}^{4} g\right\}(x)=g(x)$.

$g(x)$ 的 $s(0<|s|<2)$ 阶分数 Fourier 变换 $\left\{g^{3} g\right\}(x)$ 定义为

$$
\begin{aligned}
\{\mathscr{F} g\}(u) & =\int_{-\infty}^{\infty} B_{s}(u, x) g(x) \mathrm{d} x, \\
B_{s}(u, x) & =A_{\varphi} \exp \left[j \pi\left(u^{2} \cot (\varphi)-2 u x \csc (\varphi)+x^{2} \cot (\varphi)\right)\right], \\
A_{\varphi} & =\frac{\exp [-j(\pi \operatorname{sgn}(\sin \varphi) / 4-\varphi / 2)]}{|\sin \varphi|^{\frac{1}{2}}},
\end{aligned}
$$

其中 $\varphi=\frac{s \pi}{2}$.

利用 $\mathscr{F}^{4 k}$ ( $k$ 为任意整数) 是单位算子, 分数 Fourier 变换的定义关于阶 $s$ 可以扩展到整个 实轴 $(-\infty, \infty)$ 上. 并且由分数 Fourier 变换的定义可以得到分数 Fourier 变换算子的可加性:

$$
F^{5} \mathscr{F}^{t}=F^{\prime t} \text {, }
$$


分数 Fourier 变换算子的本征函数为 Hermite-Gaussian 函数, 即

$$
\begin{gathered}
\mathscr{F}\left\{\Psi_{n}(x)\right\}=\mathrm{e}^{-j s n \pi / 2} \Psi_{n}(u), \\
\Psi_{n}(x)=\frac{2^{1 / 4}}{\sqrt{2^{n} n !}} H_{n}(\sqrt{2 \pi} x) \exp \left(-\pi x^{2}\right),
\end{gathered}
$$

其中 $H_{n}(x)$ 是 $n$ 阶 Hermite 多项式.

分数 Fourier 变换算子的积分核可以用其本征函数展开

$$
B_{s}(u, x)=\sum_{n=0}^{\infty} \mathrm{e}^{-j s n \pi / 2} \Psi_{n}(u) \Psi_{n}(x) .
$$

\section{2 一个分数 Fourier 变换公式}

利用分数 Fourier 变换定义和 Fourier 变换的性质, 可以得到

$$
\begin{aligned}
& \int_{-\infty}^{+\infty} g^{*}\left(x-\frac{t}{2}\right) g\left(x+\frac{t}{2}\right) \exp (2 \pi j \cot (\varphi) x t) \mathrm{d} x= \\
& \operatorname{sgn}(\sin \varphi) \int_{-\infty}^{+\infty}|\{\mathscr{F} g\}(y)|^{2} \exp (2 \pi j \csc (\varphi) y t) \mathrm{d} y .
\end{aligned}
$$

事实上，

$$
\begin{aligned}
& \int_{-\infty}^{+\infty} g^{*}\left(x-\frac{t}{2}\right) g\left(x+\frac{t}{2}\right) \exp (2 \pi j \cot (\varphi) x t) \mathrm{d} x= \\
& \int_{-\infty}^{+\infty}\left\{g\left(x-\frac{t}{2}\right) \exp \left[\pi j \cot (\varphi)\left(x-\frac{t}{2}\right)^{2}\right]\right\}^{*} g\left(x+\frac{t}{2}\right) \exp \left[\pi j \cot (\varphi)\left(x+\frac{t}{2}\right)^{2}\right] \mathrm{d} x= \\
& \int_{-\infty}^{+\infty}\left\{\mathscr{F}\left[g\left(x-\frac{t}{2}\right) \exp \left(\pi j \cot (\varphi)\left(x-\frac{t}{2}\right)^{2}\right)\right](y)\right\}^{*} \mathscr{F}\left[g\left(x+\frac{t}{2}\right) \exp (\pi j \cot (\varphi)(x+\right. \\
& \left.\left.\left.\frac{t}{2}\right)^{2}\right)\right](y) \mathrm{d} y=\operatorname{sgn}(\sin \varphi) \int_{-\infty}^{+\infty}|\{\mathscr{F} g\}(y)|^{2} \exp (2 \pi j \csc (\varphi) y t) \mathrm{d} y,
\end{aligned}
$$

其中 $\mathscr{F}$ 表示 Fourier 变换.

\section{2 相位恢复问题的递推方法}

假定输入平面的波场分布是来自于波源函数 $g(x), f(u)$ 和 $g(x)$ 之间满足关系:

$$
\mathscr{F}^{a} g(x)=f(u),
$$

其中 $0<\alpha<1$. 记 $\beta=1+\alpha$, 并利用方程 (1) 和 (3), 那么可以得到

$$
\mathscr{F} g(x)=F(y) \text {. }
$$

下面只要能从 $f(u)$ 的模 $|f(u)|$ 和 $F(y)$ 的模 $|F(y)|$ 计算出函数 $g(x)$, 那么由 (7) 式, 就可求得函数 $f(u)$.

根据光学成像条件 ${ }^{[6,9]}$, 存在有限区间 $[a, b]$ 使得当 $x$ 不属于区间 $[a, b]$ 时, $g(x)=0$, 并且 $g(a) \neq 0$ 和 $g(b) \neq 0$, 那么由 (6) 式得到

$$
p(s, t)=\int_{a+\frac{t}{2}}^{b-\frac{t}{2}} g^{*}\left(x-\frac{t}{2}\right) g\left(x+\frac{t}{2}\right) \exp (2 \pi j \cot (\varphi) x t) \mathrm{d} x,
$$

其中

$$
p(s, t)=\operatorname{sgn}(\sin \varphi) \int_{-\infty}^{+\infty}|\{\mathscr{F} g\}(y)|^{2} \exp (2 \pi j \csc (\varphi) y t) \mathrm{d} y .
$$

记 $c=\frac{a+b}{2}, A(s)=\cot \varphi$ 并利用方程(9), 可得 


$$
p^{\prime}(s, b-a)=-g^{*}(a) g(b) \exp [2 \pi j A(s) c(b-a)] .
$$

根据相位分布具有平移性, 可以将 $g(a)$ 的相位指定为一定值, 比如, 取 $g(a)$ 的相位值为 0 . 下面假定它的模 $|\tilde{g}(a)|$ 为 1 , 从方程 (9) 中先求出一个特殊解 $\tilde{g}(x)$.

取 $s=\alpha$, 由(10)式可决定区间 $[a, b]$ 的长度, 使 $\tilde{g}(b) \neq 0$, 同时可计算出 $\tilde{g}(b)$ 的值. 在 区间 $[a, b]$ 上对函数 $\tilde{g}(x)$ 进行等间距采样, 可以将 $\tilde{g}(x)$ 离散为

$$
\tilde{g}(a), \tilde{g}(a+h), \tilde{g}(a+2 h), \cdots, \tilde{g}(a+n h),
$$

其中 $h=\frac{b-a}{n}$, 为了简单起见, 一般将 $n$ 取为奇数.

在(9)式中令 $t=b-a-h$, 并分别将阶 $s$ 取作 $\alpha$ 和 $\beta$, 那么利用梯形公式, 对于 $s=\alpha$ 和 $s=\beta$, 分别可将(9)式离散为

$$
\begin{aligned}
p(\alpha, b-a-h) \doteq & \frac{h}{2}\left[\tilde{g}^{*}(a+h) \tilde{g}(b) \exp \left(2 \pi j A(\alpha)\left(c+\frac{h}{2}\right)(b-a-h)\right)+\right. \\
& \left.\tilde{g}^{*}(a) \tilde{g}(b-h) \exp \left(2 \pi j A(\alpha)\left(c-\frac{h}{2}\right)(b-a-h)\right)\right]
\end{aligned}
$$

和

$$
\begin{aligned}
p(\beta, b-a-h) \doteq & \frac{h}{2}\left[\tilde{g}^{*}(a+h) \tilde{g}(b) \exp \left(2 \pi j A(\beta)\left(c+\frac{h}{2}\right)(b-a-h)\right)+\right. \\
& \left.\tilde{g}^{*}(a) \tilde{g}(b-h) \exp \left(2 \pi j A(\beta)\left(c-\frac{h}{2}\right)(b-a-h)\right)\right] .
\end{aligned}
$$

(11) 和 (12) 式关于变量 $\tilde{g}(a+h)$ 和 $\tilde{g}(b-h)$ 构成一个二元线性方程组, 通过求解方程, 可以确定 $\tilde{g}(a+h)$ 和 $\tilde{g}(b-h)$ 的值.

一般地, 在(9)式中令 $t=b-a-k h$, 并且分别取 $s=\alpha$ 和 $s=\beta$, 通过数值离散化, 可得

$$
\begin{gathered}
p(\alpha, b-a-k h)-h \sum_{i=1}^{k-1} \tilde{g}^{*}(a+\mathrm{i} h) \tilde{g}(b-k h+\mathrm{i} h) \cdot \\
\exp \left[2 \pi j A(\alpha)\left(c-\frac{k h}{2}+\mathrm{i} h\right)(b-a-k h)\right] \doteq \\
\frac{h}{2}\left\{\tilde{g}^{*}(a+k h) \tilde{g}(b) \exp \left[2 \pi j A(\alpha)\left(c+\frac{k h}{2}\right)(b-a-k h)\right]+\right. \\
\left.\tilde{g}^{*}(a) \tilde{g}(b-k h) \exp \left[2 \pi j A(\alpha)\left(c-\frac{k h}{2}\right)(b-a-k h)\right]\right\}
\end{gathered}
$$

和

$$
\begin{gathered}
p(\beta, b-a-k h)-h \sum_{i=1}^{k-1} \tilde{g}^{*}(a+\mathrm{i} h) \tilde{g}(b-k h+\mathrm{i} h) \cdot \\
\exp \left[2 \pi j A(\beta)\left(c-\frac{k h}{2}+\mathrm{i} h\right)(b-a-k h)\right] \doteq \\
\frac{h}{2}\left\{\tilde{g}^{*}(a+k h) \tilde{g}(b) \exp \left[2 \pi j A(\beta)\left(c+\frac{k h}{2}\right)(b-a-k h)\right]+\right. \\
\left.\tilde{g}^{*}(a) \tilde{g}(b-k h) \exp \left[2 \pi j A(\beta)\left(c-\frac{k h}{2}\right)(b-a-k h)\right]\right\} .
\end{gathered}
$$

求解方程 (13) 和 (14), 可得到 $\tilde{g}(a+k h)$ 和 $\tilde{g}(b-k h)$ 的值. 这样, 可递推地计算出 $\tilde{g}(a+k h)$ 的值, $k=1,2, \cdots, n$.

根据前面的推导过程, 容易得到 $g(x)$ 和 $\tilde{g}(x)$ 之间满足下列关系： 


$$
\begin{gathered}
g(a+k h)=|g(a)| * \tilde{g}(a+k h), k=1,2, \cdots, \frac{n+1}{2}, \\
g(a+k h)=\tilde{g}(a+k h) /|g(a)|, k=\frac{n+1}{2}+1, \frac{n+1}{2}+2, \cdots, n,
\end{gathered}
$$

并且直接可以证明 $g(a)$ 能够由下式确定：

$$
|g(a)|=\left\{\frac{\int_{c}^{b}|\tilde{g}(x)|^{2}}{\int_{a}^{c}|\tilde{g}(x)|^{2}}\right\}^{\frac{1}{4}} .
$$
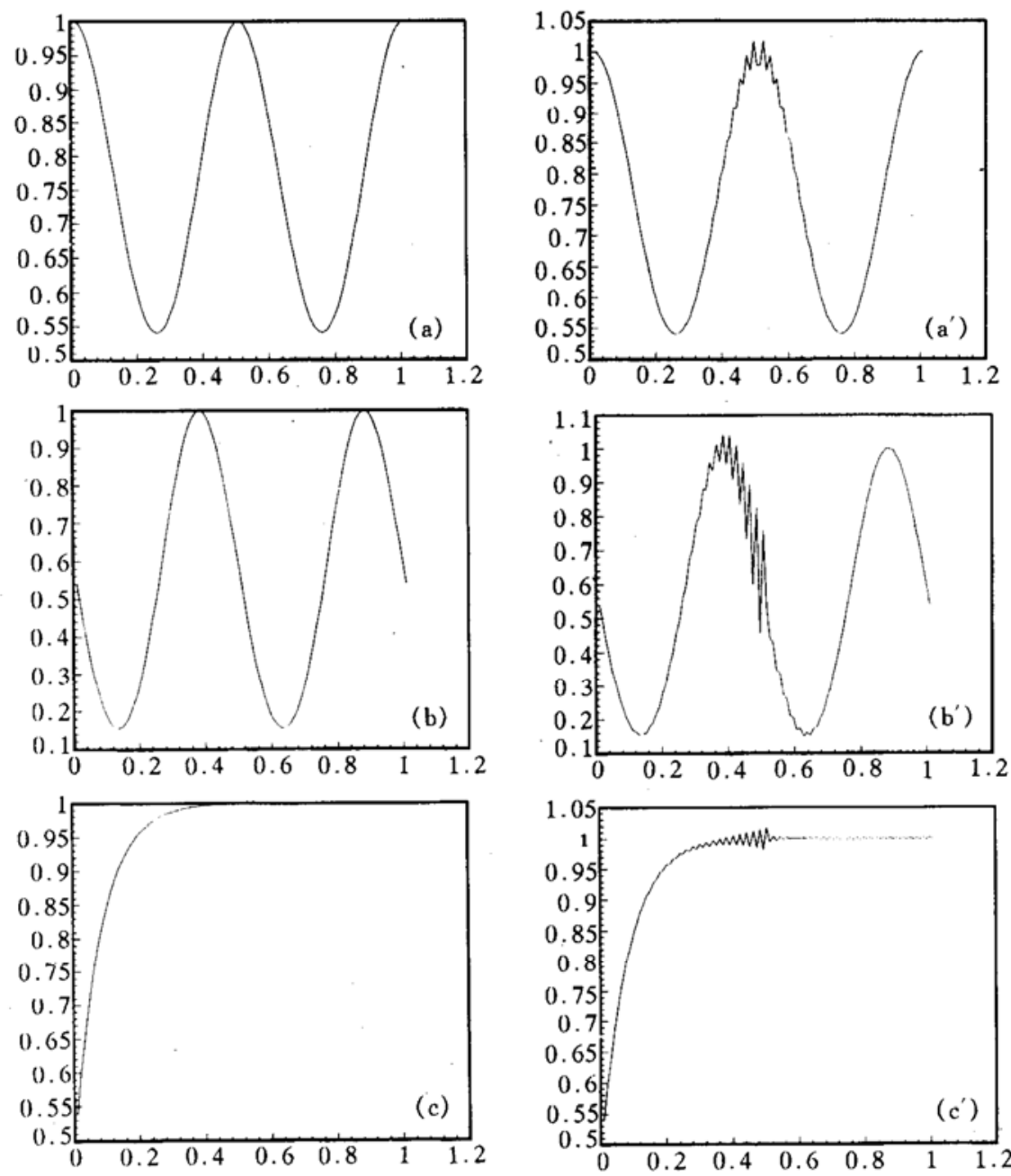

图 1 函数 $f(x)$ 与其重构函数对比曲线

\section{3 数值例子与讨论}

本节给出 3 个数值例子, 说明上节中递推方法的有效性和实用性.

( i ) $f(x)=\eta(x) \exp [j|\sin (2 \pi x)|]$;

(ii) $f(x)=\eta(x) \exp [j \sin (2 \pi x)+j \cos (2 \pi x)]$; 
( iii ) $f(x)=\eta(x) \exp [j \exp (-2 \pi x)]$, 其中

$$
\eta(x)= \begin{cases}1, & x \in[0,1], \\ 0, & x \in[0,1] .\end{cases}
$$

通过求 $f(x)$ 的 Fourier 变换 $F(u)$, 可得到它的 Fourier 变换模 $|F(u)|$. 然后通过 $f(x)$ 的模 $|f(x)|$ 和 $F(u)$ 的模 $|F(u)|$, 利用上节方法, 取 $\alpha=0.5$, 重构函数 $f(x), 3$ 个例子 ( i ), ( ii ), ( iii ) 的计算结果如图 1 所示. 在图 1 中, 图 (a) 表示函数 $f(x)=\eta(x) \exp [\mathrm{i}$. $|\sin (2 \pi x)|]$ 的实部曲线, ( $\left.\mathrm{a}^{\prime}\right)$ 表示它的重构函数的实部曲线; 图 (b) 表示函数 $f(x)=\eta(x)$. $\exp [j \sin (2 \pi x)+j \cos (2 \pi x)]$ 的实部曲线, ( $\left.\mathrm{b}^{\prime}\right)$ 表示它的重构函数的实部曲线; 图 (c) 表示函数 $f(x)=\eta(x) \exp [j \exp (-2 \pi x)]$ 的实部曲线, $\left(\mathrm{c}^{\prime}\right)$ 表示它的重构函数的实部曲线. 从图中可 看出该方法可以较好地重构函数 $f(x)$, 但随着计算步数的增多, 误差积累会对计算效果产生 一定的影响. 该方法每步求解一个二元线性方程组, 可以得到两个离散点的函数值, 与传统的 迭代方法相比, 可以大大减少计算费用, 并且不需要选择初始相位分布. 以 GS 算法为代表的 迭代方法 ${ }^{[2,3]}$, 计算效果和计算量都依赖于初始相位的选择, 有些情况下迭代过程不能收敛于 准确解. 同时本文提出的方法消除了递推过程中多解的选择问题, Dallas ${ }^{[5]}$ 和 Huiser ${ }^{[6]}$ 提出的 直接递推算法中每步递推都存在多解的选择问题. 选择真解目前还没有可靠、有效的方法,有 时使递推计算难以进行下去. 本文提出的递推算法展示了解决过去方法中存在问题的前景.

致谢 本工作为国家自然科学基金(批准号：19574010)资助项目.

\section{参考文献}

1 Baltes H P. In Inverse Source Problem in Optics. Berlin: Springer, 1978. 13 19

2 (ierchberg R W, Saxton W (). A practical algorithm for the determination of phase from image and diffraction plane pictures. Optik, 1972, 35: 237 246

3 Fienup J R. Phase retrieval algorithms: a comparison. Appl Opt, 1982, 21: $2758 \sim 2769$

4 Yang G Z, Gu B Y, Dong B Z. Theory of the amplitude-phase retrieval in an any linear transform system and its applications. Inter J Modern Phys, 1993, B7: 3 153 3224

5 Dallas W J. Digital computation of image complex amplitude from image- and diffraction-intensity: an alternative to holography. Optik, 1975, 44:45 59

6 Huiser A M J, Toorn P V, Ferwerda H A. On the problem of phase retrieval in electron microscopy from image and diffraction pattern. (Optik, 1977, 47:1 8

7 Mendlovic D, Ozaktas H M. Fractional Fourier transforms and their optical implementation 1. J Opt Soc Am, 1993, A10: $1875 \sim 1881$

8 Mendlovic D, Ozaktas H M. Fractional Fourier transforms and their optical implementation 2. J Opt Soc Am, 1993, A10: $2522 \sim 2531$

9 ()zaktas H M, Arikan (), Bozdagi G. Digital computation of the fractional Fourier transform. IEEE Transaction on Signal Processing, 1996, 44: $2141 \sim 2150$

10 Garcia J, Mas D, Dorsch R G. Fractional-Fourier-transform calculation through the fast-Fourier-transform algorithm. Appl (Opt, 1996, 35: $7013 \sim 7018$

(1997-05-05 收稿) 